\title{
Super-Resolution UWB Radar Imaging Algorithm Based on Extended Capon With Reference Signal Optimization
}

\author{
Shouhei Kidera, Associate Member, IEEE, Takuya Sakamoto, Member, IEEE, and Toru Sato, Member, IEEE
}

\begin{abstract}
Near field radar employing ultrawideband (UWB) signals with its high range resolution has great promise for various sensing applications. It enables non-contact measurement of precision devices with specular surfaces like an aircraft fuselage and wing, or a robotic sensor that can identify a human body in invisible situations. As one of the most promising radar algorithms, the range points migration (RPM) was proposed. This achieves fast and accurate surface extraction, even for complex-shaped objects, by eliminating the difficulty of connecting range points. However, in the case of a more complex shape whose variation scale is less than a pulsewidth, it still suffers from image distortion caused by multiple interference signals with different waveforms. As a substantial solution, this paper proposes a novel range extraction algorithm by extending the Capon method, known as frequency domain interferometry (FDI). This algorithm combines reference signal optimization with the original Capon method to enhance the accuracy and resolution for an observed range into which a deformed waveform model is introduced. The results obtained from numerical simulations and an experiment with bi-static extension of the RPM prove that super-resolution UWB radar imaging is accomplished by the combination between the RPM and the extended Capon methods, even for an extremely complex-surface target including edges.
\end{abstract}

Index Terms-Capon method, frequency domain interferometry (FDI), range points migration (RPM), reference signal optimization, super-resolution imaging, ultrawideband (UWB) radars.

\section{INTRODUCTION}

$\mathbf{U}$ ltrawideband (UWB) pulse radar with high range resolution has promise for near field sensing techniques. As such, it is applicable to non-contact measurement for manufacturing reflector antennas or aircraft bodies that have high-precision surfaces, or to robotic sensors that can identify a human body, even in an optically blurry vision such as dark smog in

\footnotetext{
Manuscript received March 19, 2010; revised October 12, 2010; accepted November 02, 2010. Date of publication March 03, 2011; date of current version May 04, 2011. This work was supported in part by the Grant-in-Aid for Scientific Research (A) (Grant 17206044), the Grant-in-Aid for JSPS Fellows (Grant 19-497), and in part by the Grant-in-Aid for Young Scientists (Start-up) (Grant 21860036), promoted by Japan Society for the Promotion of Science (JSPS).

S. Kidera is with the Graduate School of Informatics and Engineering, University of Electro-Communications, Tokyo, Japan (e-mail: kidera@ee.uec.ac. jp).

T. Sakamoto and T. Sato are with the Department of Communications and Computer Engineering, Graduate School of Informatics, Kyoto University, Kyoto, Japan.

Digital Object Identifier 10.1109/TAP.2011.2123059
}

disaster areas. In addition, it is suitable for surveillance or security systems for intruder detection or aged care, where an optical camera has the serious problem of privacy invasion in the case for living places. While various kinds of radar algorithms have been developed based on the aperture synthesis [1], the time reversal approach [2], [3], the range migration [4], [5] or the GA-based solution for the domain integral equations [6], they are not suitable for the above applications because of the large amount of calculation time or inadequate image resolution. To concur the problem in the conventional techniques, we have already proposed a number of radar imaging algorithms, which accomplish real-time and high resolution surface extraction beyond a pulsewidth [7], [8]. As a high-speed and accurate 3-D imaging method applicable to various target shapes, the range points migration (RPM) algorithm has been proposed [9]. This algorithm directly estimates an accurate direction of arrival (DOA) with the global characteristic of observed range points, avoiding the difficulty in connecting them. The RPM is based on a simple idea, yet, it offers an accurate target surface including the complex-shaped target that often creates an extremely complicated distribution of range points.

However, this algorithm suffers from non-negligible image distortion in the case of a more complicated target which has a surface variation less than a pulsewidth, or has many convex and concave edges. This distortion is caused by the richly interfered signals scattered from the multiple scattering centers on the target surface. These components are received within a range scale smaller than a pulsewidth, and are hardly separated by the conventional range extraction methods, such as the Wiener filter. In addition, there are small range shifts due to deformed scattered waveforms from the transmitted wave. As the conventional solution for this, the spectrum offset correction (SOC) method has been proposed [8], which directly compensates the range shift by using the center frequency offset between the scattered and transmitted waveforms. However, the SOC is rarely applied to complex-shaped targets, because it requires a completely separated waveform from other interference signals to calculate an accurate center frequency.

To overcome this difficulty, this paper proposes a novel range extraction algorithm by extending the frequency domain Capon method. While the Capon is useful for enhancing the range resolution based on FDI [10]-[12], the resolution and accuracy of this method significantly depend on a reference waveform such as the transmitted waveform. In general, the scattered waveform from the target with a wavelength scale differs from the one transmitted [8], and the range resolution given by the original 


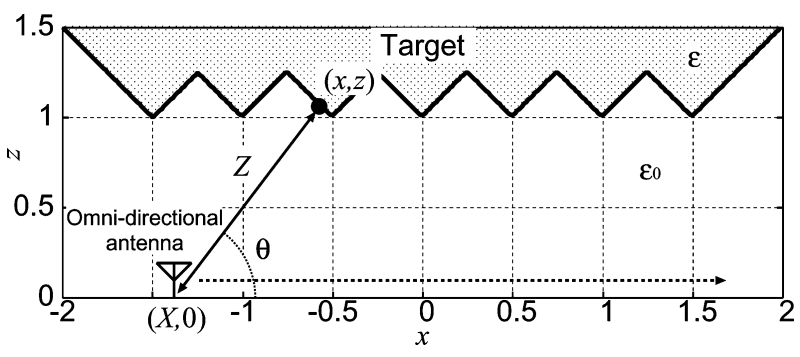

Fig. 1. System model in the 2-dimensional model.

Capon method distorts due to this deformation. To outperform the original Capon, this paper extends the original Capon so that it optimizes the reference signal based on the simplified waveform model. The extended Capon significantly enhances the range resolution and accuracy, and brings out the utmost performance of the RPM algorithm. The results obtained from numerical simulations in the 2-dimensional (2-D) and 3-dimensional (3-D) models are presented in Sections II and III. Finally, an experiment using the UWB module, where the bi-static extension of the RPM is applied, verifies that super-resolution imaging is accomplished by the combination between the RPM and the extended Capon, when using a simple radar constitution.

\section{2-D PROBLEM}

\section{A. System Model}

Fig. 1 shows the system model in the 2-D model. It assumes the mono-static radar, and an omni-directional antenna is scanned along the $x$-axis. It is assumed that the target has an arbitrary shape with a clear boundary. The propagation speed of the radio wave $c$ is assumed to be a known constant. A mono-cycle pulse is used as the transmitting current. The real space in which the target and antenna are located, is expressed by the parameters $(x, z)$. The parameters are normalized by $\lambda$, which is the central wavelength of the pulse. $z>0$ is assumed for simplicity. $s^{\prime}\left(X, Z^{\prime}\right)$ is defined as the received electric field at the antenna location $(x, z)=(X, 0)$, where $Z^{\prime}=c t /(2 \lambda)$ is a function of time $t$.

\section{B. RPM Algorithm}

Various kinds of radar imaging algorithms based on an aperture synthesis, time reversal or range migration methods, have been proposed [1]-[6]. As a real-time imaging algorithm, the SEABED has been developed, which uses a reversible transform boundary scattering transform (BST) between the observed ranges and the target boundary [7]. In addition, another high-speed imaging algorithm termed Envelope has been developed aiming at improving the image stability of SEABED, by avoiding the range derivative operations [8]. While these algorithms accomplish fast and accurate imaging for a simple shaped object, such as trapezoid, pyramid, or sphere shapes, it is hardly applicable to complex-shaped or multiple targets because it requires correct connection of range points.

As one of the most promising algorithms applicable to various target shapes, the RPM algorithm has been proposed [9]. This method assumes that a target boundary point $(x, z)$ exists on a circle with center $(X, 0)$ and radius $Z$, and then employs an
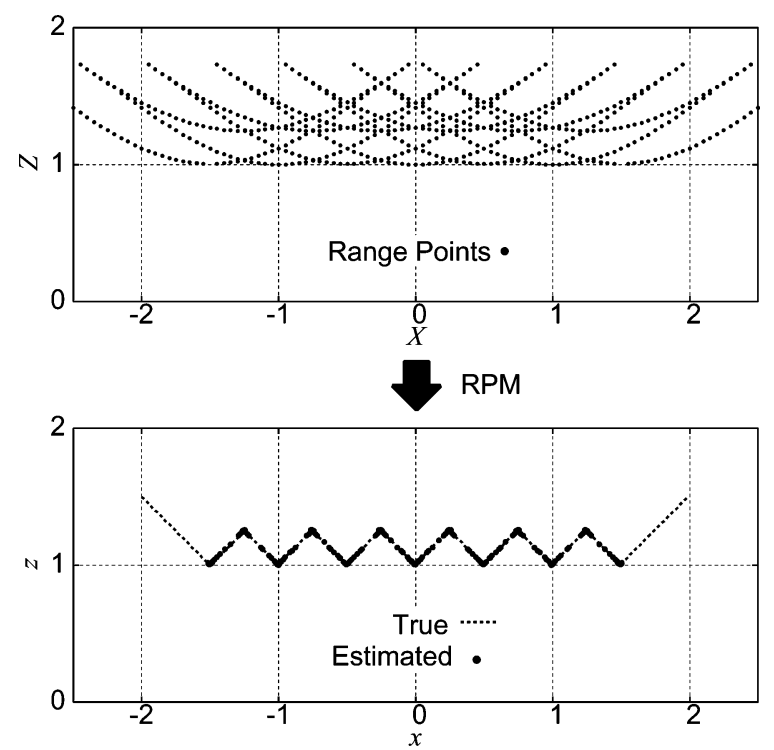

Fig. 2. True range points (upper) and extracted target points with the RPM (lower).

accurate DOA (Direction of Arrival $\theta$ in Fig. 1) estimation by making use of the global characteristics of the observed range map. The optimum $\theta_{\text {opt }}$ is calculated as,

$$
\begin{aligned}
\theta_{\text {opt }}(\boldsymbol{q})= & \arg \max _{0 \leq \theta \leq \pi} \\
& \left|\sum_{i=1}^{N_{q}} s\left(\boldsymbol{q}_{i}\right) e^{-\left\{\left(X-X_{i}\right)^{2} / 2 \sigma_{X}^{2}+\left(\theta-\theta\left(\boldsymbol{q}, \boldsymbol{q}_{i}\right)\right)^{2} / 2 \sigma_{\theta}^{2}\right\}}\right|
\end{aligned}
$$

where $\boldsymbol{q}=(X, Z), \boldsymbol{q}_{i}=\left(X_{i}, Z_{i}\right)$ and $s(\boldsymbol{q})$ denotes the amplitude of the received signal at the range $Z$ and the antenna location $(X, 0) . N_{q}$ is the number of the range points. $\theta\left(\boldsymbol{q}, \boldsymbol{q}_{\boldsymbol{i}}\right)$ denotes the angle from the $x$ axis to the intersection point of the circles, with parameters $(X, Z)$ and $\left(X_{i}, Z_{i}\right)$. The constants $\sigma_{\theta}$ and $\sigma_{X}$ are empirically determined. The detail of this algorithm is described in [9]. The target boundary $(x, z)$ for each range point $(X, Z)$ is expressed as $x=X+Z \cos \theta_{\text {opt }}(\boldsymbol{q})$ and $z=Z \sin \theta_{\text {opt }}(\boldsymbol{q})$. This algorithm ignores range points connection, and produces accurate target points, even if extremely complicated range distribution is given. Thus, the inaccuracy occurring in the SEABED and Envelope can be substantially avoided by this method.

Fig. 2 shows the example of this algorithm under the assumption that the true range points are given as in the upper side of this figure. Here, $s(\boldsymbol{q})=1.0$ for every $\boldsymbol{q}$ is set for simplicity. The lower side of Fig. 2 shows a distinct advantage for this algorithm in that it can accurately locate the target points, even if a quite complicated range map is given.

\section{Performance of RPM Using the Wiener Filter}

The performance example of the RPM is presented here, where the received electric field is calculated by the FDTD (Finite Difference Time Domain) method. The former study [9] employs the Wiener filter in order to extract a range point for each location. The range points $(X, Z)$ are extracted from the local peaks of $s\left(X, Z^{\prime}\right)$ which are beyond the determined 


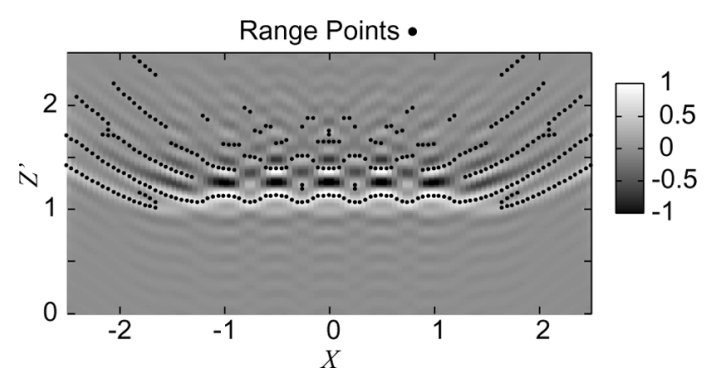

Fig. 3. Output of the Wiener filter and extracted range points.

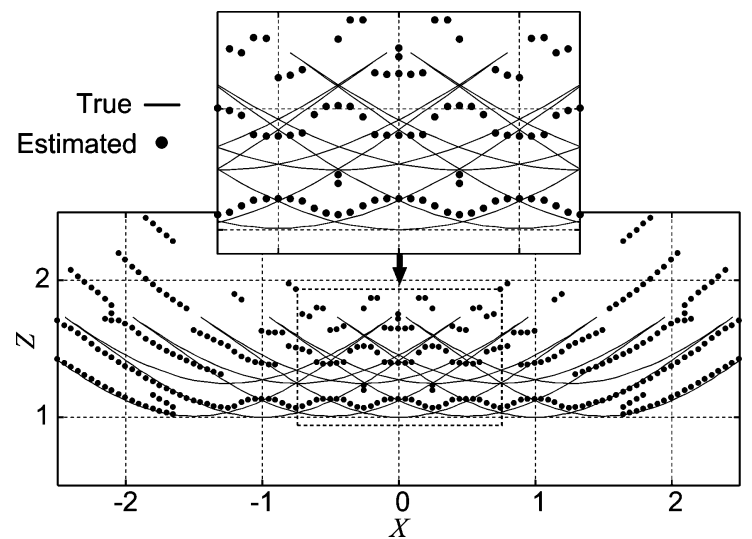

Fig. 4. Estimated target points with the RPM and the Wiener filter.

threshold. The procedure is detailed in [9]. The example of this method for the target shape shown in Fig. 1 is presented. Fig. 3 shows the output of the Wiener filter, and the extracted range points. The received signals are calculated at 101 locations between $-2.5 \leq X \leq 2.5$. A noiseless environment is assumed. Fig. 4 presents the comparison between the true and extracted range points in this case. It shows that the range points suffer from the inaccuracy due to the multiple interference echoes within a range scale of less than a pulsewidth. Fig. 5 shows the target points, when the RPM is applied to the range points in Fig. 4. This figure indicates that the inaccuracy of range points distorts the target image, which is totally inadequate for identifying its actual shape, especially for convex and concave edges. Furthermore, this range map includes a small error caused by scattered waveform deformation, whose characteristics are detailed in [13].

To enhance the accuracy for range points extraction, the spectrum offset correction (SOC) algorithm has been developed aiming at compensating the range shift due to the waveform deformation [8]. It is, however, confirmed that the range accuracy of the SOC is entirely inadequate in such a richly interfered situation. This is because the range errors in this case are dominantly caused by the peak shift of the output of the Wiener filter due to the interferences of multiple scattering echoes. Furthermore, the SOC is based on the center periods estimation of the scattered signal, when each signal should be correctly resolved in the time domain. This is, however, difficult when the multiple interfered signals are mixed together in a time scale less than its center period.

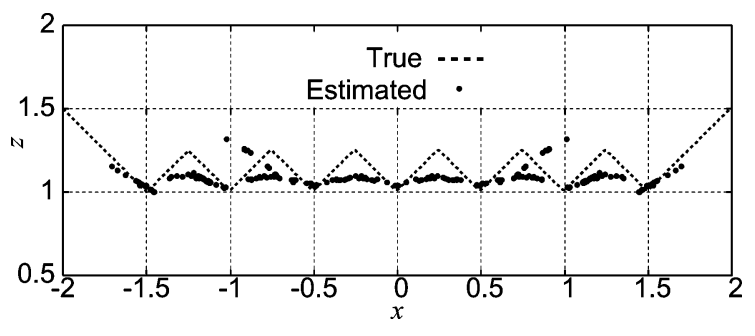

Fig. 5. Estimated target points with the RPM and the Wiener filter.

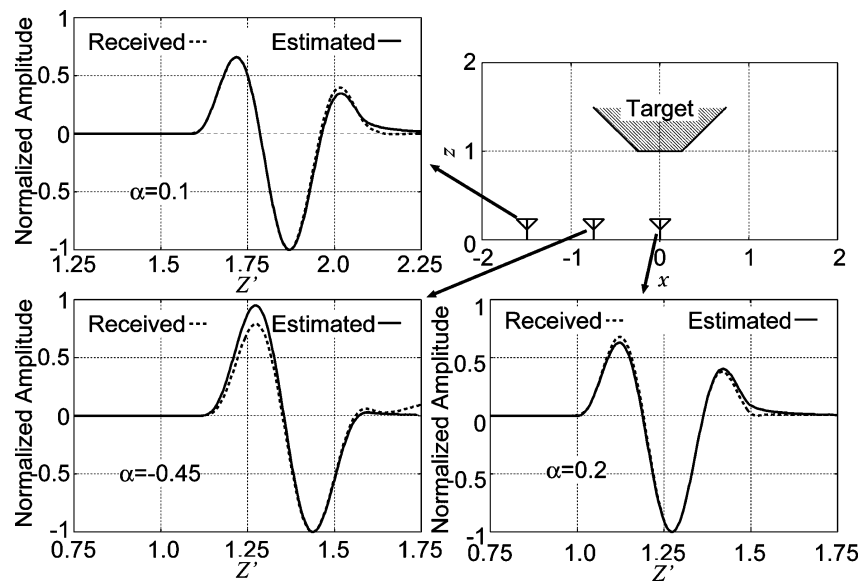

Fig. 6. Waveform comparisons for each antenna location at polygonal target.

\section{Proposed Range Extraction Algorithm}

To overcome the difficulty described above, this paper proposes a novel algorithm for range points extraction by extending the frequency domain Capon method. The Capon algorithm is one of the most powerful tools for enhancing range resolution based on FDI. It is confirmed, however, that the scattered waveform deformation distorts the range resolution and accuracy of the original Capon method. As a solution for this, the proposed method optimizes the reference signal used in the Capon. This method introduces a reference waveform model, based on the fractional derivative of the transmitted waveform as

$$
S_{\mathrm{ref}}(\omega, \alpha)=(j \omega)^{\alpha} S_{\mathrm{tr}}(\omega)^{*}
$$

where $S_{\mathrm{tr}}(\omega)$ is the angular frequency domain of the transmitted signal and ${ }^{*}$ denotes a complex conjugate. $\alpha$ is a variable which satisfies $|\alpha| \leq 1$.

The waveform comparison using this simplified model is demonstrated as follows. Fig. 6 shows the scattered waveform from the polygonal target received at the different locations, and the estimated waveforms with the optimized $\alpha$ in (2). This figure indicates that a scattered waveform differs depending on an antenna location, or a local shape around the scattering center [13]. This deformation distorts the resolution and accuracy of the original Capon method, because it employs a phase and amplitude interferometry in each frequency between the reference and scattered waveforms. It is confirmed that an estimated waveform with the optimized $\alpha$ in the previous model accurately approximates an actual deformed waveform, 
where the range accuracy is estimated of the order of $0.01 \lambda$ when using the matched filter.

Based on this waveform model, the observed vector $\boldsymbol{V}_{n}(\alpha, L)$ is defined as

$$
\boldsymbol{V}_{n}(\alpha, \boldsymbol{L})=\left[\frac{S\left(\omega_{n}, \boldsymbol{L}\right)}{S_{\mathrm{ref}}\left(\omega_{n}, \alpha\right)}, \ldots, \frac{S\left(\omega_{n+M-1}, \boldsymbol{L}\right)}{S_{\mathrm{ref}}\left(\omega_{n+M-1}, \alpha\right)}\right]^{T}
$$

where $S(\omega, L)$ denotes the received signal in angular frequency domain at each antenna location $\boldsymbol{L}=(X, 0)$, and $M$ denotes the dimension of $\boldsymbol{V}_{n}(\alpha, \boldsymbol{L})$. Here, in order to suppress a range sidelobe caused by the coherent interference signals, frequency averaging is used. The averaged correlation matrix $\boldsymbol{R}(\alpha, \boldsymbol{L})$ is defined as

$$
\boldsymbol{R}(\alpha, \boldsymbol{L})=\sum_{n=1}^{N-M+1} \boldsymbol{V}_{n}(\alpha, \boldsymbol{L}) \boldsymbol{V}_{n}^{H}(\alpha, \boldsymbol{L})
$$

where $H$ denotes the Hermitian transpose. $N$ is the total number of the frequency points, and is determined by the maximum frequency band of the transmitted signal. $M \leq N$ holds. The output of the extended Capon $s_{\mathrm{cp}}\left(\alpha, Z^{\prime}, L\right)$ is defined as

$$
s_{\mathrm{cp}}\left(\alpha, Z^{\prime}, \boldsymbol{L}\right)=\frac{S_{0}^{-1}}{\boldsymbol{a}^{H}\left(Z^{\prime}\right) \boldsymbol{R}(\alpha, \boldsymbol{L})^{-1} \boldsymbol{a}\left(Z^{\prime}\right)}
$$

where $a\left(Z^{\prime}\right)$ denotes the steering vector of $Z^{\prime}$ for each frequency

$$
\boldsymbol{a}\left(Z^{\prime}\right)=\left[e^{-j \omega_{1} 2 Z^{\prime} \lambda / c}, e^{-j \omega_{2} 2 Z^{\prime} \lambda / c}, \ldots, e^{-j \omega_{M} 2 Z^{\prime} \lambda / c}\right]^{T}
$$

$S_{0}$ is defined as

$$
S_{0}=\sqrt{\int\left\{\boldsymbol{a}^{H}\left(Z^{\prime}\right) \boldsymbol{R}(\alpha, L)^{-1} \boldsymbol{a}\left(Z^{\prime}\right)\right\}^{-2} d Z^{\prime}} .
$$

The normalization with $S_{0}$ enables us to compare the amplitude of $s_{\mathrm{cp}}\left(\alpha, Z^{\prime}, L\right)$ with respect to $\alpha$. Then, the local maximum of $s_{\mathrm{cp}}\left(\alpha, Z^{\prime}, \boldsymbol{L}\right)$ for $\alpha$ and $Z^{\prime}$ offers an optimized range resolution in the Capon method. That is, $s_{\mathrm{cp}}\left(\alpha, Z^{\prime}, \boldsymbol{L}\right)$ performs the highest range resolution, where the reference waveform most coincides with the actual scattered waveform. Finally, it determines the range points $(X, Z)$, which satisfies the following conditions

$$
\left.\begin{array}{c}
\frac{\partial s_{\mathrm{cp}}\left(\alpha, Z^{\prime}, \boldsymbol{L}\right)}{\partial \alpha}=0 \\
\frac{\partial s_{\mathrm{cp}}\left(\alpha, Z^{\prime}, \boldsymbol{L}\right)}{\partial Z^{\prime}}=0 \\
s_{\mathrm{cp}}\left(\alpha, Z^{\prime}, \boldsymbol{L}\right) \geq \beta \max _{Z^{\prime}} s_{\mathrm{cp}}\left(\alpha, Z^{\prime}, \boldsymbol{L}\right)
\end{array}\right\} .
$$

where $\beta=0.3$ is empirically determined according to the evaluations in [9]. Equation (8) is numerically solved by searching the local maxima of $s_{\mathrm{cp}}\left(\alpha, Z^{\prime}, L\right)$ for $\alpha$ and $Z^{\prime}$. This algorithm selects an accurate range point by enhancing the range resolution of the Capon method with the optimized reference signal. Each target point $(x, z)$ is calculated from the group of range points in (1), that is the RPM. This extraction algorithm achieves accurate range point estimation by compensating for the waveform deformation. This is a distinct advantage compared with the original Capon method.

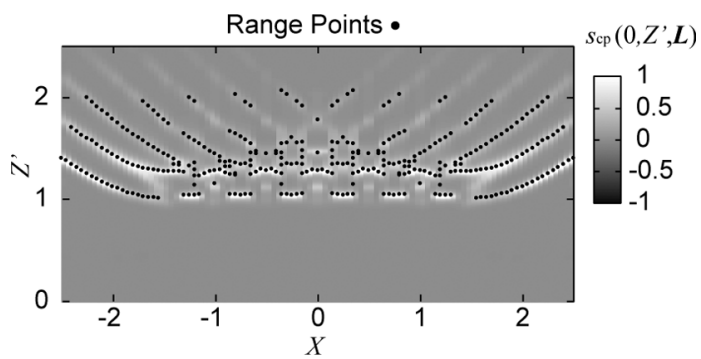

Fig. 7. Output of the original Capon method and extracted range points.

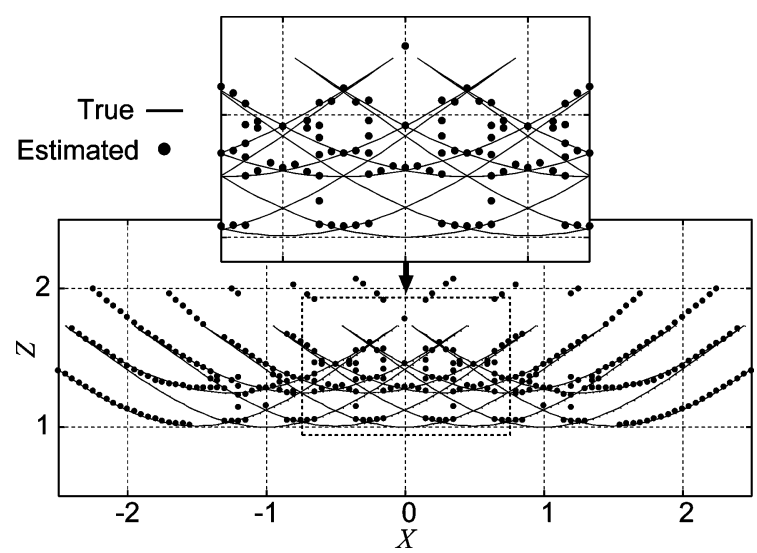

Fig. 8. Estimated range points with the original Capon method.

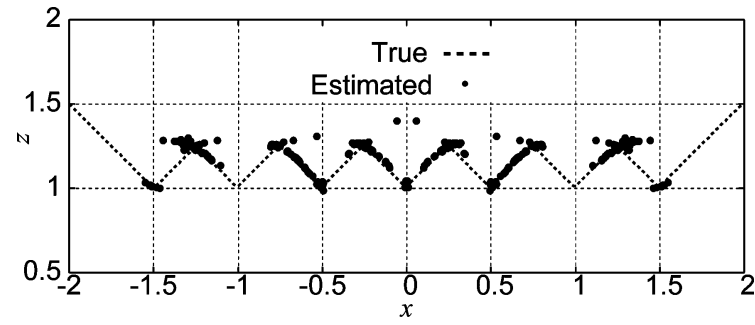

Fig. 9. Estimated target points with the RPM and the the original Capon method.

\section{E. Performance Evaluation in Numerical Simulation}

This section presents the examples for each range extraction method, where the same target as in Fig. 1 is assumed. Here, $N=60$ and $M=20$ are set. Fig. 7 shows the output of the original Capon method and the extracted range points, which corresponds to $\alpha=0$ in (8), that is, the waveform deformation is not considered. Fig. 8 shows the comparison between the true and extracted range points in this case. In this figure, the number of the accurate range points increases compared to Fig. 4, because the original Capon enhances the range resolution. Fig. 9 shows the estimated target points by using the original Capon method as in Fig. 8. This figure also shows that it enhances the accuracy of the location of imaging points, and the convex edge region is also accurately located. However, the inaccuracy around the concave edge region is recognized, and some parts of the target boundary are still not reconstructed. This is because of the distorted resolution and accuracy of ranges caused by the reference and actual scattered waveform being in-coincidence. 


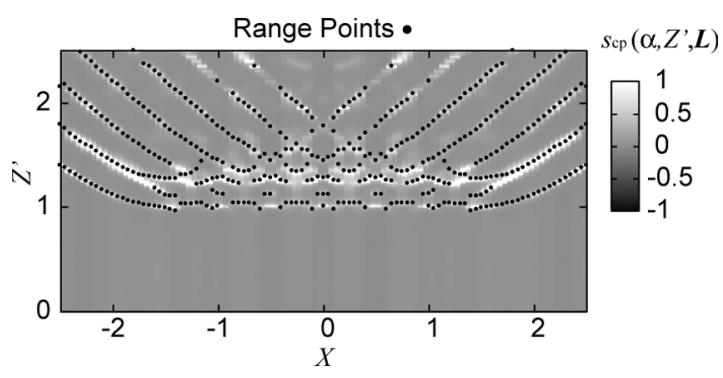

Fig. 10. Output of the extended Capon method and extracted range points.

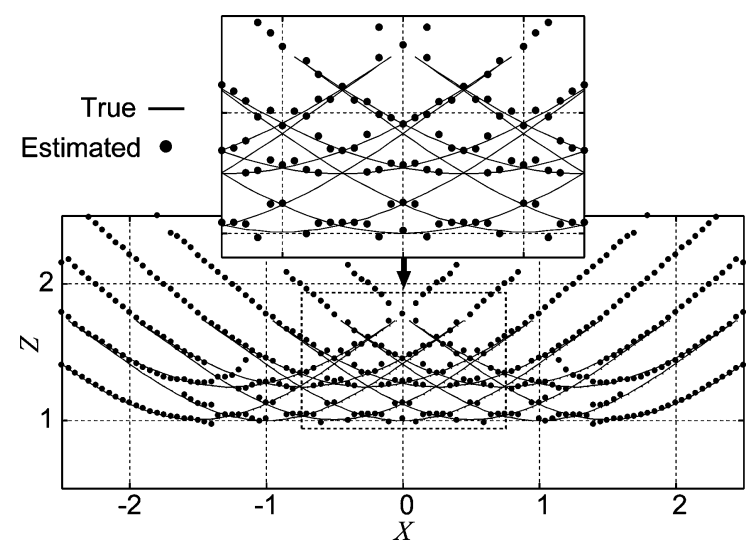

Fig. 11. Estimated range points with the extended Capon method.

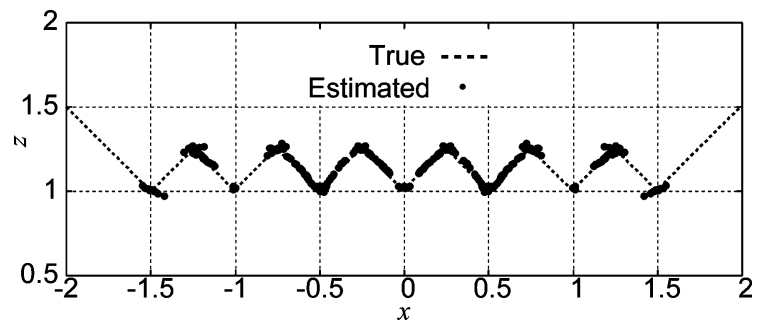

Fig. 12. Estimated target points with the RPM and the extended Capon method.

In contrast, Fig. 10 shows $s_{\mathrm{cp}}\left(\alpha, Z^{\prime}, \boldsymbol{L}\right)$ with the optimized $\alpha$, and the range points extracted. Fig. 11 offers the same view as in Fig. 8. $N$ and $M$ and are the same as in the previous example. This figure verifies that the extracted range points are accurately located, and the number of those points increases compared with the original Capon method. Fig. 12 shows the estimated target points obtained by the RPM. This figure shows these points accurately reconstruct the convex or concave edge region, and offer substantial information for identifying the complicated target shape, even with convex or concave edges. This is because the proposed method enhances the resolution of $s_{\mathrm{cp}}\left(\alpha, Z^{\prime}, \boldsymbol{L}\right)$ with respect to the scattered waveform deformation. Thereby, the peaks, which are regarded as the trivial value in the output of the original Capon, can be detected by optimizing the reference waveform.

As the comparison for the other methods not specified to the clear boundary extraction, the SAR (Synthetic Aperture Radar) method is introduced. This algorithm is the most useful tool for radar imaging [1], and the near field extension of the SAR is applied here [9]. Fig. 13 shows the example of the SAR. While the image produced by the SAR is stable, its spatial resolution

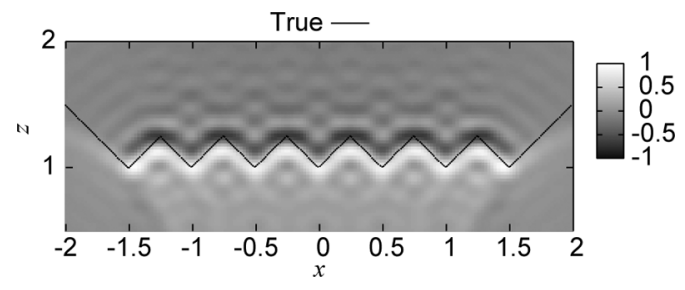

Fig. 13. Estimated image with the synthetic aperture radar (SAR).

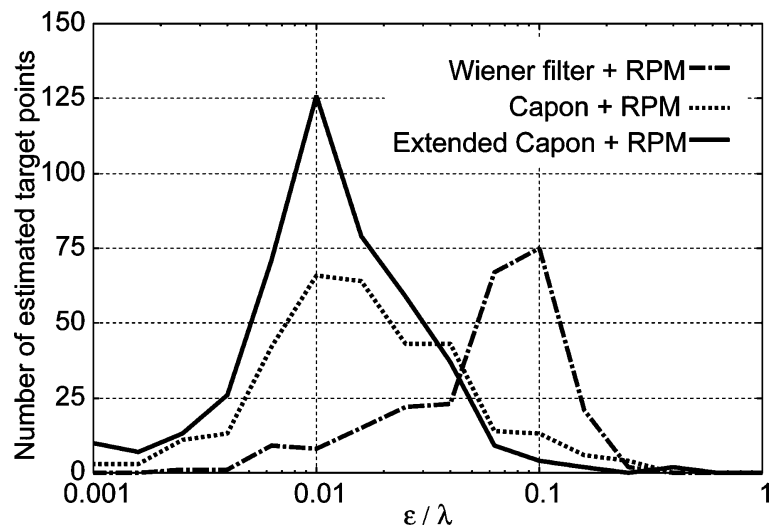

Fig. 14. Number of the target points for each $\epsilon$.

determined by a half of the pulsewidth is substantially inadequate for recognizing the concave or convex edges. This result also proves the advantage for the proposed method, in terms of high-resolution imaging.

Here, the quantitative analysis is introduced by $\epsilon$ defined as

$$
\epsilon_{i}=\min _{\boldsymbol{p}_{\text {true }}}\left\|\boldsymbol{p}_{\text {true }}-\boldsymbol{p}_{e}^{i}\right\|, \quad\left(i=1,2, \ldots, N_{T}\right)
$$

where $\boldsymbol{p}_{\text {true }}$ and $\boldsymbol{p}_{e}^{i}$ express the locations of the true and estimated target points, respectively. $N_{T}$ is the total number of $\boldsymbol{p}_{e}^{i}$. Fig. 14 plots the number of the estimated points for each value of $\epsilon$. This figure verifies that the number of the accurate target points with the proposed method significantly increases around $0.01 \lambda$, compared with other conventional algorithms. $\bar{\epsilon}$, that is defined as the mean values of $\epsilon_{i}$, for each method is $5.66 \times 10^{-2} \lambda$ for the Wiener filter, $2.18 \times 10^{-2} \lambda$ for the original Capon, and $1.23 \times 10^{-2} \lambda$ for the proposed extended Capon, respectively. This result quantitatively proves the effectiveness of the proposed range extraction algorithm.

In addition, the examples in noisy situation are investigated, where the white Gaussian noise is added to each received signal as $s^{\prime}\left(X, Z^{\prime}\right)$. Fig. 15 shows the estimated points with the RPM and the extended Capon, where the mean $\mathrm{S} / \mathrm{N}$ is $35 \mathrm{~dB}$. The $\mathrm{S} / \mathrm{N}$ is defined as the ratio of peak instantaneous signal power to the averaged noise power after applying the matched filter with the transmitted waveform. This figure shows that the target points around the convex edge region are scarcely extracted, and the accuracy of the points near the concave edges is distorted. This is because this algorithm uses the inverse filtering in creating the observed vector $\boldsymbol{V}_{n}(\alpha, \boldsymbol{L})$ in (3), which is sensitive to the white noise. Furthermore, the scattered signals from the convex edges are relatively smaller than those from the concave boundaries. Then, the significant range peaks around the convex region are 


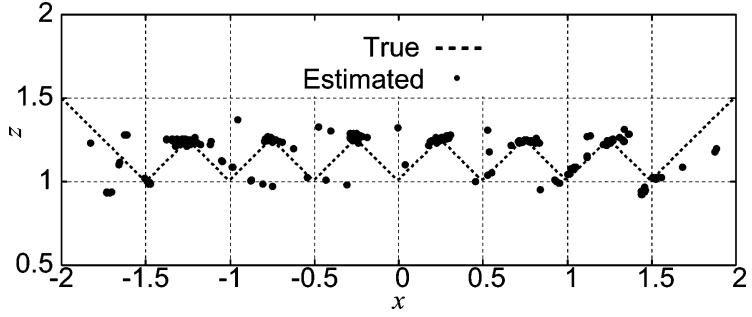

Fig. 15. Estimated target points with the RPM and the extended Capon method in $\mathrm{S} / \mathrm{N}=35 \mathrm{~dB}$.

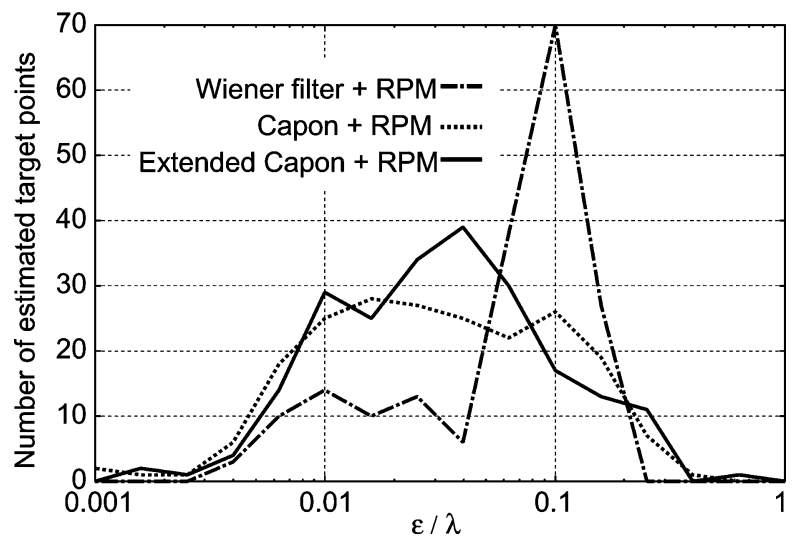

Fig. 16. Number of the target points for each $\epsilon$ in $\mathrm{S} / \mathrm{N}=35 \mathrm{~dB}$.

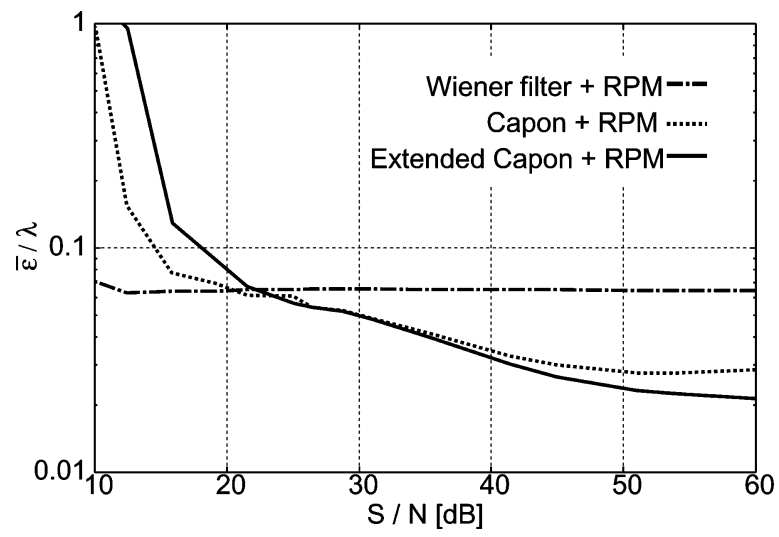

Fig. 17. Relationship between $\bar{\epsilon}$ and $\mathrm{S} / \mathrm{N}$ for each method.

not detected in this case. Fig. 16 plots the number of the estimated points for each value of $\epsilon$ in this noisy case. $\bar{\epsilon}$ for each method are $6.00 \times 10^{-2} \lambda$ for the Wiener filter, $4.37 \times 10^{-2} \lambda$ for the original Capon, and $4.44 \times 10^{-2} \lambda$ for the extended Capon. While the accuracy for the both original and extended Capon methods deteriorates due to the noises, the superiority to the Wiener filter is maintained. This is because the range resolution of the Wiener filter also becomes more inadequate than that in the noiseless case. In addition, it is a substantial characteristic of the proposed method that the higher $\mathrm{S} / \mathrm{N}$ provides the higher resolution for the obtained images.

Fig. 17 illustrates the relationship between $\bar{\epsilon}$ and $\mathrm{S} / \mathrm{N}$ for each method. This figure shows that, in the case of $\mathrm{S} / \mathrm{N} \geq 30 \mathrm{~dB}$, the

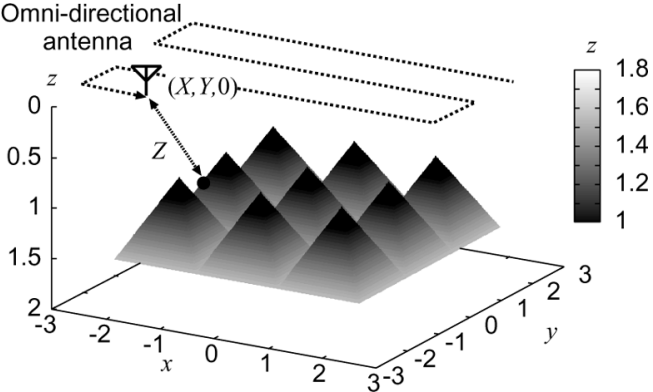

Fig. 18. System model in 3-dimensional problem.

proposed method accomplishes most accurate target imaging. While this method requires a high $\mathrm{S} / \mathrm{N}$ to hold the accuracy, the actual UWB radar system can achieve this level of S/N. This is because we assume the near field measurement, where each receiver obtains an intensive echo from objects even under the spectrum mask of the UWB signal [14], and random noises in received signals can be considerably suppressed using coherent averaging. In contrast, in the case of $\mathrm{S} / \mathrm{N} \leq 22 \mathrm{~dB}$, the conventional Wiener filter holds its accuracy within $0.08 \lambda$, while the both original and extended Capon methods deteriorate their accuracies over $0.1 \lambda$. This is because these methods are based on the inverse filtering in creating the observation subvector in (3). It is our future work to enhance the accuracy in the noisy situation to modify the definition of $\boldsymbol{V}_{n}(\alpha, \boldsymbol{L})$ regarding to the $\mathrm{S} / \mathrm{N}$.

Moreover, the proposed method employs an optimization process for each received signal and requires around $100 \mathrm{sec}$ for the range points extraction, whereas the algorithm with the original Capon method requires only $5 \mathrm{sec}$. We need to select an appropriate range extraction method for the different kinds of applications, whether they require real-time operation or extremely accurate surface extraction in the high $\mathrm{S} / \mathrm{N}$.

\section{3-D PROBLEM}

\section{A. System Model}

Fig. 18 shows the system model in the 3-D problem. The target model, antenna, and transmitted signal are the same as those assumed in the 2-D problem. The antenna is scanned on the plane, $z=0$. It assumes a linear polarization in the direction of the $x$-axis. $R$-space is expressed by the parameter $(x, y, z)$. We assume $z \geq 0$ for simplicity. $s^{\prime}\left(X, Y, Z^{\prime}\right)$ is defined as the received electric field at the antenna location $(x, y, z)=$ $(X, Y, 0)$.

\section{B. Extension of Proposed Method to 3-D Model}

The extension of the RPM to the 3-D model has been derived in [9]. This assumes that a target boundary point $(x, y, z)$ exists on a sphere with center $(X, Y, 0)$ and radius $Z$. It calculates $(x, y, z)$ by investigating the distribution of the intersection circles between the spheres determined with $(X, Y, Z)$ and $\left(X_{i}, Y_{i}, Z_{i}\right)$. Each intersection circle projected to the $z=$ 


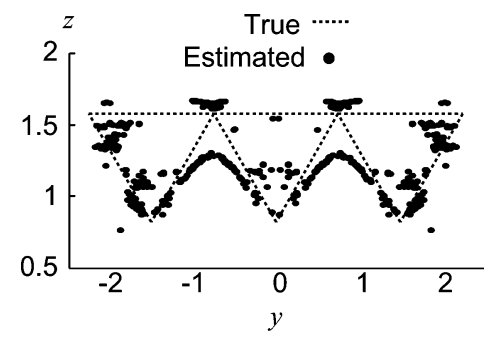

Fig. 19. Estimated target points with the RPM and the Wiener filter for $-0.75 \leq x \leq 0.75$.

0 plane becomes a straight line, which is defined as $L_{i}$. This method determines the target location $(x, y)$ as

$$
\begin{aligned}
& \left(x\left(\boldsymbol{q}^{3 d}\right), y\left(\boldsymbol{q}^{3 d}\right)\right)=\arg \max _{x, y} \\
& \left|\sum_{i=1}^{N_{q}} s\left(\boldsymbol{q}_{i}\right) \exp \left\{-\frac{d\left(x, y, \boldsymbol{q}^{3 d}, \boldsymbol{q}_{i}^{3 d}\right)^{2}}{2 \sigma_{d}^{2}}-\frac{D\left(\boldsymbol{q}^{3 d}, \boldsymbol{q}_{i}^{3 d}\right)^{2}}{2 \sigma_{D}^{2}}\right\}\right|
\end{aligned}
$$

where $\boldsymbol{q}^{3 d}=(X, Y, Z), \boldsymbol{q}_{i}^{3 d}=\left(X_{i}, Y_{i}, Z_{i}\right)$, and $s(\boldsymbol{q})$ denotes the amplitude of the received signal at the range $Z$ and the antenna location $(X, Y, 0) . d\left(x, y, \boldsymbol{q}^{3 d}, \boldsymbol{q}_{i}^{3 d}\right)$ denotes the minimum distance between the line $L_{i}$ and $(x, y, 0) . D\left(\boldsymbol{q}^{3 d}, \boldsymbol{q}_{i}^{3 d}\right)=\sqrt{\left(X-X_{i}^{2}\right)+\left(Y-Y_{i}^{2}\right)}, \sigma_{d}$ and $\sigma_{D}$ are empirically determined. Under the assumption $z \geq 0$, the $z$ coordinate of each target point is given by $z\left(\boldsymbol{q}^{3 d}\right)=\sqrt{Z^{2}-\left\{x\left(\boldsymbol{q}^{3 d}\right)-X\right\}^{2}-\left\{y\left(\boldsymbol{q}^{3 d}\right)-Y\right\}^{2}}$. This algorithm ignores the connecting procedures of a large number of range points, and can avoid instability due to the failure of range connections. Thus, it produces accurate target points, even if an extremely complicated 3-D range map is given. The detailed characteristic is described in [9].

Furthermore, in the proposed range extraction, each range point $(X, Y, Z)$ is calculated in (8), where $\boldsymbol{L}=(X, Y, 0)$ is redefined. Each target point $(x, y, z)$ is calculated from the group of range points in (10).

\section{Performance Evaluation in Numerical Simulation}

This section presents an example of the proposed method using numerical simulation. The mono-static radar is scanned for $-2.5 \leq x, y \leq 2.5$, where the number of locations on each axis is 51. The target boundary is assumed as in Fig. 18. Fig. 19 shows the target points obtained by the RPM in the use of the Wiener filter for range extraction at $-0.75 \leq x \leq 0.75$. $\sigma_{d}=0.1 \lambda$ and $\sigma_{D}=0.6 \lambda$ are set. This figure shows that the estimated target points suffer from severe inaccuracy, and the produced target boundary is far from the actual one. This is because the obtained image with the RPM seriously depends on the accuracy for the range points, which have non-negligible errors due to multiple interfered signals in the same range gate, or scattered waveform deformation [13].

In contrast, Fig. 20 shows the estimated target points with the RPM and the original Capon method, where $\alpha=0$ is set in (8). Here, $N=100$ and $M=20$ are set. This figure proves that accurate target points estimation is achieved only by enhancing the range resolution. However, it should be noted that the target

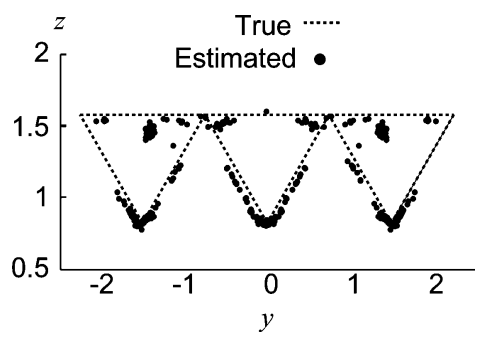

Fig. 20. Estimated target points with the RPM and the original Capon method for $-0.75 \leq x \leq 0.75$.

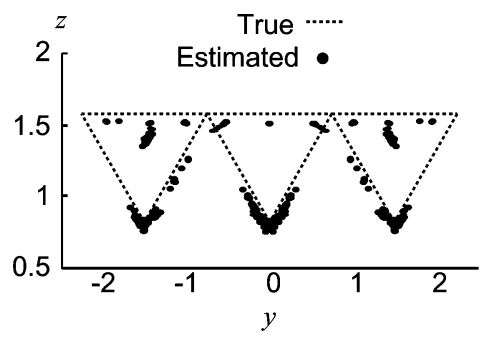

Fig. 21. Estimated target points with the RPM and the extended Capon method for $-0.75 \leq x \leq 0.75$.

points around the convex edge region are still inaccurate, because this method does not consider the waveform deformation which distorts the resolution and accuracy in range extraction.

Fig. 21 shows the same view as in Fig. 19, when the extended Capon method is used for range extraction. $N$ and $M$ are the same as in the previous example. It is confirmed that this method improves the accuracy for target point extraction, especially for the edge region. This is because the range resolution of the Capon is significantly enhanced by optimizing the reference waveform. Here, the deep-set concave region is not reconstructed, because the direct scattered signals from this region are not received at any antenna location. This is an inherent problem under the assumption that a single scattered signal is used for imaging. Another study offers a promising solution for this problem by using the multiple scattered waves for imaging [15].

In addition, Fig. 22 shows the number of the target points for each sampled $\epsilon_{i}$, which is defined in (9). This figure proves that the proposed method enhances the number of the accurate target points around $\epsilon=0.04 \lambda . \bar{\epsilon}$ for each method are $0.070 \lambda$ for the Wiener filter, $0.044 \lambda$ for the original Capon, and $0.035 \lambda$ for the extended Capon. The RPM with the original Capon creates a few target points with $\epsilon \geq 0.3 \lambda$, and these points increase $\bar{\epsilon}$ compared with that of the proposed method, even if the same $\beta$ in (8) is used. This is because the extended Capon method avoids the peak lowering around the actual ranges in $s\left(\alpha, X, Z^{\prime}\right)$ with the optimized $\alpha$.

\section{Performance Evaluation in Experiment}

This section investigates the experimental study of the proposed algorithm. We utilize a UWB pulse with a center frequency of $3.3 \mathrm{GHz}$ and a $10 \mathrm{~dB}$-bandwidth of $3.0 \mathrm{GHz}$. The center wavelength $\lambda$ of the pulse is $91 \mathrm{~mm}$. The antenna has an elliptic polarization, of which the ratio of major to minor axes is about $17 \mathrm{~dB}$, and the direction of the polarimetry axis 


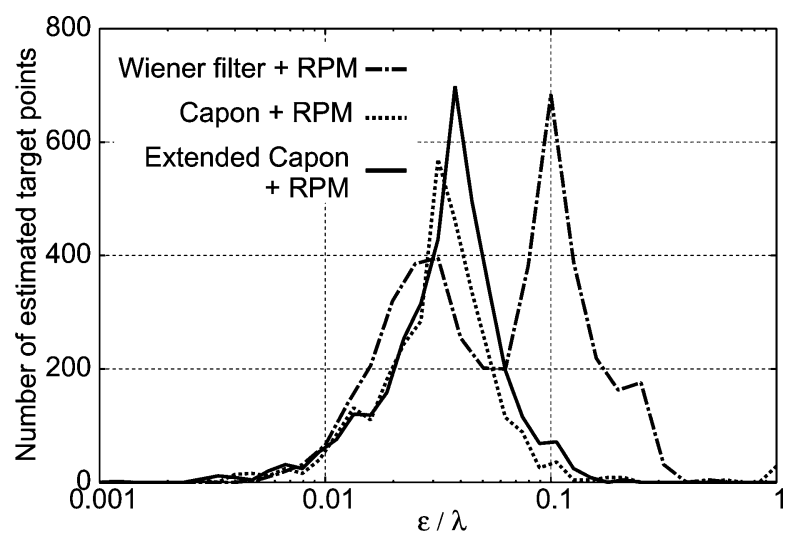

Fig. 22. Number of the target points for each $\epsilon$.

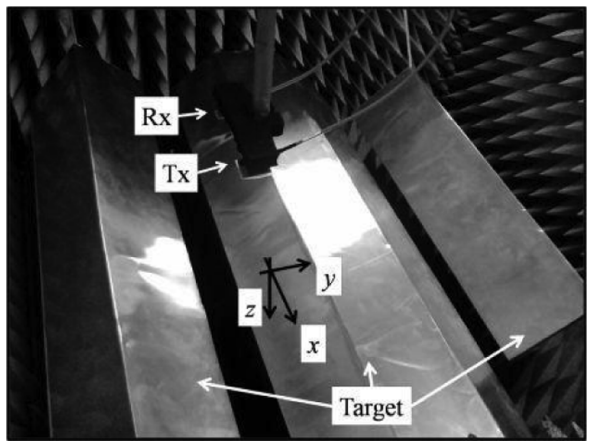

Fig. 23. Arrangement for the multiple targets and the small UWB microstrip patch antenna.

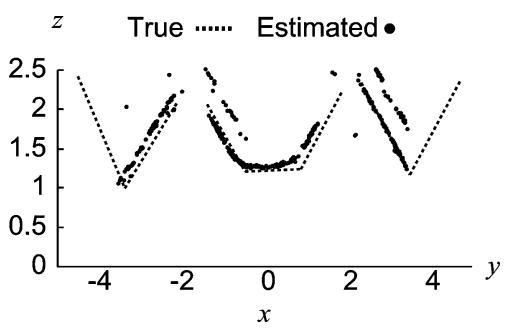

Fig. 24. Estimated target points with the RPM and the Wiener filter in the experiment for $-0.5 \leq y \leq 0.5$.

of the antenna is along the $y$-axis. The $3 \mathrm{~dB}$-beamwidth of the antenna is about $90^{\circ}$. One trapezoid and two triangle prismatic targets are set, and are covered with $0.2 \mathrm{~mm}$ thick aluminum sheet. Fig. 23 illustrates the arrangement of antennas with respect to the multiple targets. The transmitting and receiving antennas are scanned on the $z=0$ plane, for $-3.3 \leq x \leq 3.3$ and $-1.1 \leq y \leq 1.1$, respectively, with both sampling intervals set to $0.11 \lambda$. The separation between the transmitting and receiving antennas is $1.4 \lambda$ in the $y$-direction. The data are coherently averaged 1024 times. The direct scattered signals from the targets are obtained by eliminating the direct signal from the transmitting antenna.

Fig. 24 shows the extracted target points using the RPM for $-0.5 \leq y \leq 0.5$, when the Wiener filter is used for range extraction. The mean $\mathrm{S} / \mathrm{N}$ is around $30 \mathrm{~dB}$. Here, the bi-static extension of RPM is applied, which is detailed in Appendix A.

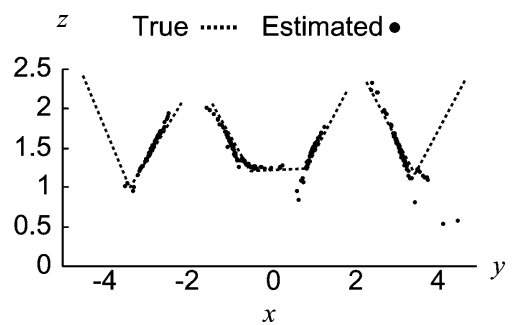

Fig. 25. Estimated target points with the RPM and the original Capon in the experiment for $-0.5 \leq y \leq 0.5$.

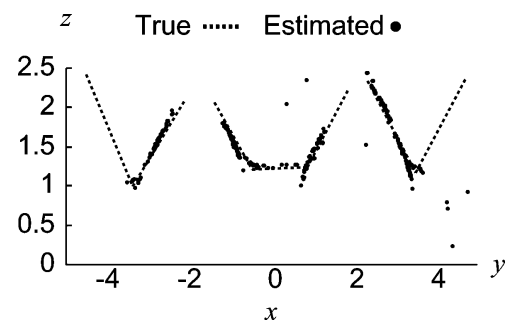

Fig. 26. Estimated target points with the RPM and the extended Capon in the experiment for $-0.5 \leq y \leq 0.5$.

$\sigma_{d}=0.1 \lambda$ and $\sigma_{D}=0.6 \lambda$ are set. This figure proves that the target points have non-negligible errors, especially around the trapezoid edge region. In addition, the unnecessary image appears above the actual boundary due to the range sidelobe of the Wiener filter. Fig. 25 shows the same view as in Fig. 24, when the original Capon method is applied to the same received data. $N=50$ and $M=10$ are set. In this figure, it is confirmed that the target points accurately express an actual target surface, including the edge region, and the false image due to the range sidelobe in the Wiener filter is considerably suppressed. This proves that the frequency domain interferometry in the Capon method is sufficiently effective in terms of accurate surface extraction. Furthermore, Fig. 26 shows the same view as in Fig. 24, when the extended Capon is applied for range extraction. While the image obtained using the proposed method creates an accurate target boundary including edges, there is not a significant difference between the images with the both original and extended Capon.

Fig. 27 shows the number of the target points with the sampled $\epsilon_{i}$ defined in (9) for each method. The methods with the original and extended Capon obtain an increase in the number of points with the accuracy around $0.04 \lambda . \bar{\epsilon}$ for each method are $1.49 \times 10^{-1} \lambda$ for the Wiener filter, $4.53 \times 10^{-2} \lambda$ for the original Capon, and $5.55 \times 10^{-2} \lambda$ for the extended Capon. The effectiveness of the extended Capon is not obvious in this case. This is because this experiment does not offer the sufficient $\mathrm{S} / \mathrm{N}$ to recognize the significant discrepancies between the original and extended Capon. According to the discussion in Section II-E, the higher $\mathrm{S} / \mathrm{N}$ is required to confirm the effectiveness of the extended Capon, and this is an inherent characteristic of this method. However, the both Capon methods accomplish accurate imaging including edge region by suppressing the false images 


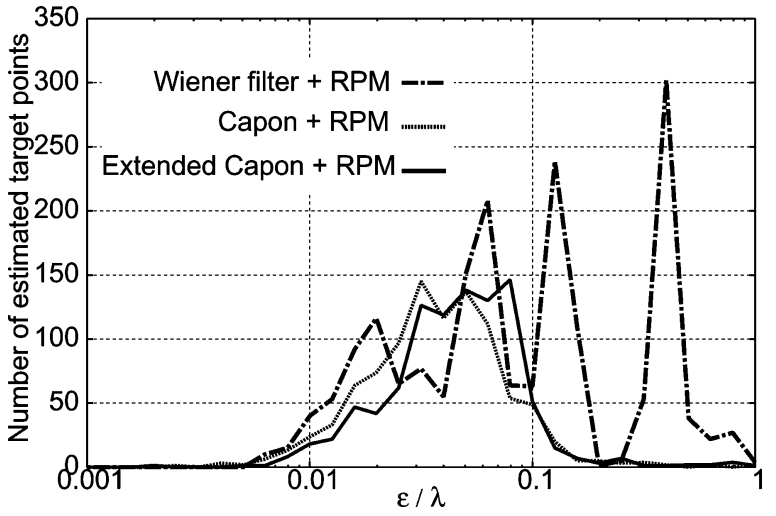

Fig. 27. Number of the target points for each $\epsilon$ in the experiment.

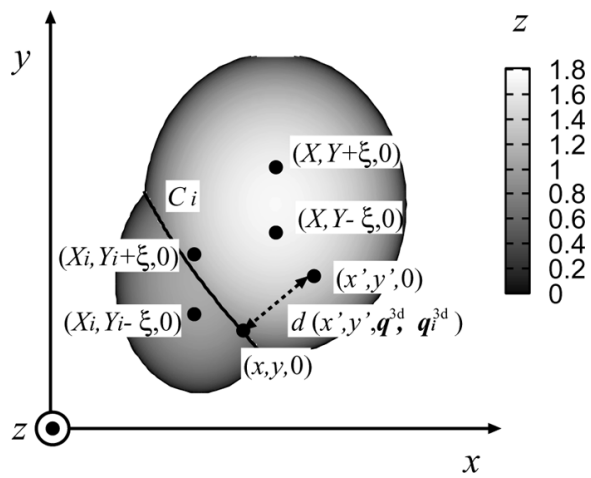

Fig. 28. Intersection curve $C_{i}$ between two ellipsoids determined by $(X, Y, Z)$ and $\left(X_{i}, Y_{i}, Z_{i}\right)$.

due to the range sidelobe, which are not avoided by the Wiener filter.

\section{CONCLUSION}

This paper proposed a novel range extraction algorithm as the extended frequency domain Capon method, known as FDI. To enhance the image quality of the RPM method, including the case for complicated shaped objects with concave or convex edges, this method extends the original Capon so that it optimizes the reference signal with a simplified and accurate waveform model. It has a substantial advantage that the range resolution is remarkably enhanced, even if the different scattered waves are mixed together within the range scale less than a pulsewidth. The results from numerical simulations verified that the extended Capon method created accurate range points of the order of $0.01 \lambda$, where the RPM offered an utmost performance and achieved super-resolution imaging even for the complex-shaped 3-D objects. Finally, in the experiment employing the UWB module, the RPM with the bi-static extension and the extended Capon method significantly improved the accuracy of target surface extraction including the edge boundaries. These results prove that the proposed method has a great potential for super-resolution radar imaging with a non-parametric approach.

\section{APPENDIX \\ BISTATIC EXTENSION OF RPM}

The bi-static extension of RPM in 3-dimensional model is derived here. At the experimental model assuming in this paper, the transmitting and receiving antenna locations are defined as $(X, Y+\xi, 0)$ and $(X, Y-\xi, 0)$, where $\xi$ is a constant. In the modified RPM algorithm, $d\left(x^{\prime}, y^{\prime}, \boldsymbol{q}^{3 d}, \boldsymbol{q}_{i}^{3 d}\right)$ is defined as the minimum distance between $\left(x^{\prime}, y^{\prime}, 0\right)$ and the curve $C_{i}$, which is projected on $z=0$ plane from the intersection curve between the following two ellipsoids with $\boldsymbol{q}^{3 d}$ and $\boldsymbol{q}_{i}^{3 d}$

$$
\begin{aligned}
& \frac{(x-X)^{2}}{Z^{2}-\xi^{2}}+\frac{(y-Y)^{2}}{Z^{2}}+\frac{z^{2}}{Z^{2}-\xi^{2}}=1 \\
& \frac{\left(x-X_{i}\right)^{2}}{Z_{i}^{2}-\xi^{2}}+\frac{\left(y-Y_{i}\right)^{2}}{Z_{i}^{2}}+\frac{z^{2}}{Z_{i}^{2}-\xi^{2}}=1 .
\end{aligned}
$$

$C_{i}$ is formulated as

$$
\begin{aligned}
& -2\left(X-X_{i}\right) x+\frac{\xi^{2}}{Z^{2} Z_{i}^{2}}\left(Z^{2}-Z_{i}^{2}\right) y^{2} \\
& -2\left\{\left(Y-Y_{i}\right)-\xi^{2}\left(\frac{Y}{Z^{2}}-\frac{Y_{i}}{Z_{i}^{2}}\right)\right\} y \\
& +X^{2}-X_{i}^{2}+Y^{2}-Y_{i}^{2}-\left(Z^{2}-Z_{i}^{2}\right) \\
& -\xi^{2}\left(\frac{Y^{2}}{Z^{2}}-\frac{Y_{i}^{2}}{Z_{i}^{2}}\right)=0 .
\end{aligned}
$$

In corresponding to the left term in (12), $f(x, y)$ is defined as,

$$
f(x, y) \equiv A x+B y^{2}+C y+D
$$

where

$$
\left.\begin{array}{ccc}
A= & -2\left(X-X_{i}\right) \\
B= & \frac{\xi^{2}}{Z^{2} Z_{i}^{2}}\left(Z^{2}-Z_{i}^{2}\right) \\
C= & -\xi^{2}\left(\frac{Y}{Z^{2}}-\frac{Y_{i}}{Z_{i}^{2}}\right) \\
D= & \left.-\left(Z^{2}-Z_{i}^{2}\right)-\xi^{2}\left(\frac{Y^{2}}{Z^{2}}-\frac{Y_{i}^{2}}{Z_{i}^{2}}\right)\right)
\end{array}\right\} .
$$

The point $(x, y)$, which minimizes the distance to the curve $C_{i}$, is calculated by solving the constrained optimization problem as

$$
\left.\begin{array}{cc}
\text { minimize } & \left(x-x^{\prime}\right)^{2}+\left(y-y^{\prime}\right)^{2} \\
\text { subject to } & f(x, y)=0
\end{array}\right\} .
$$

$L(x, y)$ is defined with the Lagrange multiplier $\nu$ as

$$
L(x, y)=\left(x-x^{\prime}\right)^{2}+\left(y-y^{\prime}\right)^{2}+\nu f(x, y)
$$

Then, $(x, y)$ satisfies the following conditions

$$
\left.\begin{array}{l}
f(x, y)=0 \\
\frac{\partial L(x, y)}{\partial x}=0 \\
\frac{\partial L(x, y)}{\partial y}=0
\end{array}\right\} .
$$


Solving these equations, the following cubic equation of $\nu$ is derived,

$$
\begin{aligned}
A^{2} B^{2} \nu^{3} & \\
& -\left\{\frac{2 A B\left(x^{\prime}-A\right)+B\left(4 B D-C^{2}\right)}{2}\right\} \nu^{2} \\
& -\left\{4\left(x^{\prime} A B+B D\right)-\left(A^{2}+C^{2}\right)\right\} \nu-2 f\left(x^{\prime}, y^{\prime}\right) \\
= & 0 .
\end{aligned}
$$

With the solution $\nu_{\mathrm{sol}}$ in (18), the minimum distance is calculated as

$$
d\left(x^{\prime}, y^{\prime}, \boldsymbol{q}^{d}, \boldsymbol{q}_{i}^{3 d}\right)=\frac{1}{2} \sqrt{\left(\nu_{\mathrm{sol}} x^{\prime}\right)^{2}+\left\{\frac{\nu_{\mathrm{sol}}\left(C+2 y^{\prime} B\right)}{\left(1+B \nu_{\mathrm{sol}}\right)}\right\}^{2}} .
$$

This derivation is readily extended to the general bi-static or multistatic model by modifying the definition of the ellipsoids in (11).

\section{REFERENCES}

[1] D. L. Mensa, G. Heidbreder, and G. Wade, "Aperture synthesis by object rotation in coherent imaging," IEEE Trans. Nucl. Sci., vol. 27, no. 2, pp. 989-998, Apr. 1980.

[2] D. Liu, G. Kang, L. Li, Y. Chen, S. Vasudevan, W. Joines, Q. H. Liu, J. Krolik, and L. Carin, "Electromagnetic time-reversal imaging of a target in a cluttered environment," IEEE Trans. Antenna Propag., vol. 53, no. 9, pp. 3058-3066, Sep. 2005.

[3] D. Liu, J. Krolik, and L. Carin, "Electromagnetic target detection in uncertain media: Time-reversal and minimum-variance algorithms," IEEE Trans. Geosci. Remote Sens., vol. 45, no. 4, pp. 934-944, Apr. 2007.

[4] J. Song, Q. H. Liu, P. Torrione, and L. Collins, "Two-dimensional and three dimensional NUFFT migration method for landmine detection using ground-penetrating radar," IEEE Trans. Geosci. Remote Sens., vol. 44, no. 6, pp. 1462-1469, Jun. 2006.

[5] F. Soldovieri, A. Brancaccio, G. Prisco, G. Leone, and R. Pieri, "A kirchhoff-based shape reconstruction algorithm for the multimonostatic configuration: The realistic case of buried pipes," IEEE Trans. Geosci. Remote Sens., vol. 46, no. 10, pp. 3031-3038, Oct. 2008.

[6] A. Massa, D. Franceschini, G. Franceschini, M. Pastorino, M. Raffetto, and M. Donelli, "Parallel GA-based approach for microwave imaging applications," IEEE Trans. Antenna Propag., vol. 53, no. 10, pp. 3118-3127, Oct. 2005.

[7] T. Sakamoto and T. Sato, "A target shape estimation algorithm for pulse radar systems based on boundary scattering transform," IEICE Trans. Commun., vol. E87-B, no. 5, pp. 1357-1365, 2004.

[8] S. Kidera, T. Sakamoto, and T. Sato, "High-resolution and real-time UWB radar imaging algorithm with direct waveform compensations," IEEE Trans. Geosci. Remote Sens., vol. 46, no. 11, pp. 3503-3513, Nov. 2008.

[9] S. Kidera, T. Sakamoto, and T. Sato, "Accurate UWB radar 3-D imaging algorithm for complex boundary without range points connections," IEEE Trans. Geosci. Remote Sens., vol. 48, no. 4, pp. 1993-2004, Apr. 2010.

[10] J. Capon, "High-resolution frequency-wavenumber spectrum analysis," Proc. IEEE, vol. 57, no. 8, pp. 1408-1418, Aug. 1969.
[11] J. Capon and N. R. Goodman, "Probability distributions for estimators of frequency wavenumber spectrum," Proc. IEEE, vol. 58, no. 10, pp. 1785-1786, Oct. 1970.

[12] C. D. Richmond, "Capon algorithm mean-square error threshold SNR prediction and probability of resolution," IEEE Trans. Signal Processing, vol. 53, no. 8, pp. 2748-2764, Aug. 2005.

[13] S. Kidera, T. Sakamoto, and T. Sato, "A high-resolution imaging algorithm without derivatives based on waveform estimation for UWB radars," IEICE Trans. Commun., vol. E90-B, no. 6, pp. 1487-1494, Jun. 2007.

[14] Federal Communications Commission (FCC), Office of Engineering and Technology (OET) Technology (OET) Bulletin No. 65, Aug. 1997, no. Supplement C, pp. 35-35.

[15] S. Kidera, T. Sakamoto, and T. Sato, "Experimental study of shadow region imaging algorithm with multiple scattered waves for UWB radars," Proc. PIERS, vol. 5, no. 4, pp. 393-396, Aug. 2009.

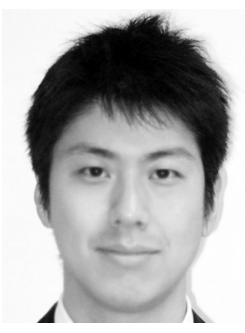

Shouhei Kidera (A'08) received the B.E. degree in electrical and electronic engineering and the M.I. and $\mathrm{Ph}$.D. degrees in informatics from Kyoto University, Kyoto, Japan, in 2003, 2005, and 2007, respectively.

$\mathrm{He}$ is an Assistant Professor in the Graduate School of Informatics and Engineering, University of Electro-Communications, Japan. His current research interest is in advanced signal processing for the near field radar, UWB radar.

Dr. Kidera is a member of the Institute of Electronics, Information, and Communication Engineers of Japan (IEICE) and the Institute of Electrical Engineering of Japan (IEEJ).

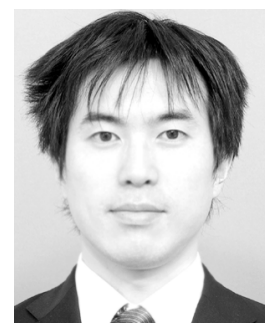

Takuya Sakamoto (M’04) was born in Nara, Japan, in 1977. He received the B.E., M.I., and Ph.D. degrees from Kyoto University, Kyoto, Japan, in 2000, 2002, and 2005, respectively.

$\mathrm{He}$ is an Assistant Professor in the Department of Communications and Computer Engineering, Graduate School of Informatics, Kyoto University. His current research interest is in signal processing for UWB pulse radars.

Dr. Sakamoto is a member of the Institute of Electronics, Information, and Communication Engineers of Japan (IEICE), and the Institute of Electrical Engineering of Japan (IEEJ).

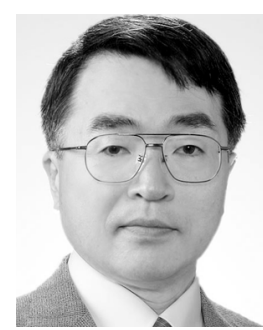

Toru Sato (M'92) received the B.E., M.E., and Ph.D. degrees in electrical engineering from Kyoto University, Kyoto, Japan, in 1976, 1978, and 1982, respectively.

He has been with Kyoto University since 1983 and is currently a Professor in the Department of Communications and Computer Engineering, Graduate School of Informatics. His major research interests have been system design and signal processing aspects of atmospheric radars, radar remote sensing of the atmosphere, observations of precipitation using radar and satellite signals, radar observation of space debris, and imaging with UWB pulse radars.

Dr. Sato was awarded the Tanakadate Prize in 1986. He is a fellow of the Institute of Electronics, Information, and Communication Engineers of Japan, and a member of the Society of Geomagnetism and Earth, Planetary and Space Sciences, the Japan Society for Aeronautical and Space Sciences, and the American Meteorological Society. 